Journal of Engineering and Applied Sciences 14 (Special Issue 1): 3842-3844, 2019

ISSN: 1816-949X

(C) Medwell Journals, 2019

\title{
Fuzzy Neural Network in Circumstance Maintanence for Marine Electric Propulsion System
}

\author{
D.T. Jacob Jai Kumar \\ Department of Marine Engineering, AMET University, Chennai, India
}

\begin{abstract}
Since, marine electric drive framework is made out of various types of gear with confused yet interconnected structure it ought to apply condition support to unravel upkeep not on time or over-upkeep. Connected condition support could enhance the utilization of types of gear in order to enhance operational proficiency and upkeep capacity of ship. Perceiving the deficiencies of non-insight of fluffy hypothesis and additionally moderate merging of canny neural-arrange, the Fluffy Neural system (FNN) strategy for condition support of marine electric drive framework is connected in the study. It represented the superiority of FNN method and using FNN to set up the progress and implement steps of condition maintenance of marine electric propulsion system. Using the certain ship's measurement data we obtained a model of condition maintenance model with FNN. Simulation using MATLAB shows that it can provide high precision and is suitable for project application.
\end{abstract}

Key words: Marine, electric propulsion system, condition maintenance, fuzzy neural network, MATLAB, FNN

\section{INTRODUCTION}

With the rapid development of electron semiconductor technology and $\mathrm{AC}$ speed regulation theory as well as microcomputer technology marine electric propulsion system had huge evolutions in mobility and reliability and running efficiency and propulsion power and so on. ship electric propulsion system is explained by Bao and Shi (2011). The electric propulsion application fields are enlarged and show wide market foreground.

The keen strategies in view of information are applying computerized reasoning speculations and techniques in CM. CBM of transmission and transformation equipment is discussed by Feng et al. (2012). It is anything but difficult to apply in venture about $\mathrm{CM}$ by embracing status data and legitimate smart number juggling to assess framework and give advices. Wise assessment number juggling is the key of research in $\mathrm{CM}$ innovation. Condition assessment for power transformer is described by Chengguang and Wenwen (2012). It connected fuzzy comprehensive evaluation hypothesis on electric transformer. However, the assessment comes about precision wasn't high on the grounds that fuzzy comprehensive theory hasn't learning capacity and the fluffy connection lattice was hard to been fabricated. Bridge technical state based on the BP neural network is described by Musheng et al. (2011). BP neural network strategy to assess the status of scaffold. In any case, the preparation of neural system was finished through a large number of iterative, so, speed was ease back to impact the ongoing execution of assessment. Decision-making model for condition assessment of power transformers using fuzzy approach and evidential reasoning and mathematical programming are discussed by Liao et al. (2011), Lai and Hwang (2012).

\section{MATERIALS AND METHODS}

Contents: Commonly used intelligent methods mainly had ANN and FT which have own characters compared in Table 1. Fuzzy Neural Network (FNN) combines fuzzy theory and ANN to form better system than individual Fuzzy system or ANN system. Ship trajectory control using particle swarm optimizatiovn is explained by Sethuramalingam and Nagaraj (2016). FNN is suitable to express certain or fuzzy knowledge and its inference seem as human's thinking pattern to process the uncertain non-liner problems. FNN is advantaged to

Table 1: the comparison about ANN with FT

\begin{tabular}{lll}
\hline Comparision & ANN & Fuzzy theory \\
Structure & $\begin{array}{l}\text { Network interlinked } \\
\text { with neural cells } \\
\text { Parallel processing, } \\
\text { tolerance and self-study } \\
\text { ability }\end{array}$ & $\begin{array}{l}\text { Fuzzy rules and } \\
\text { fuzzy inference } \\
\text { Procession2 uncertain } \\
\text { information experience } \\
\text { and knowledge, } \\
\text { physics concept clear }\end{array}$ \\
& $\begin{array}{l}\text { Difficult to understand } \\
\text { knowledge, slow } \\
\text { convergence, local } \\
\text { disficult to setup } \\
\text { optimization }\end{array}$ & $\begin{array}{l}\text { relation matrix, low } \\
\text { precision }\end{array}$ \\
\hline
\end{tabular}




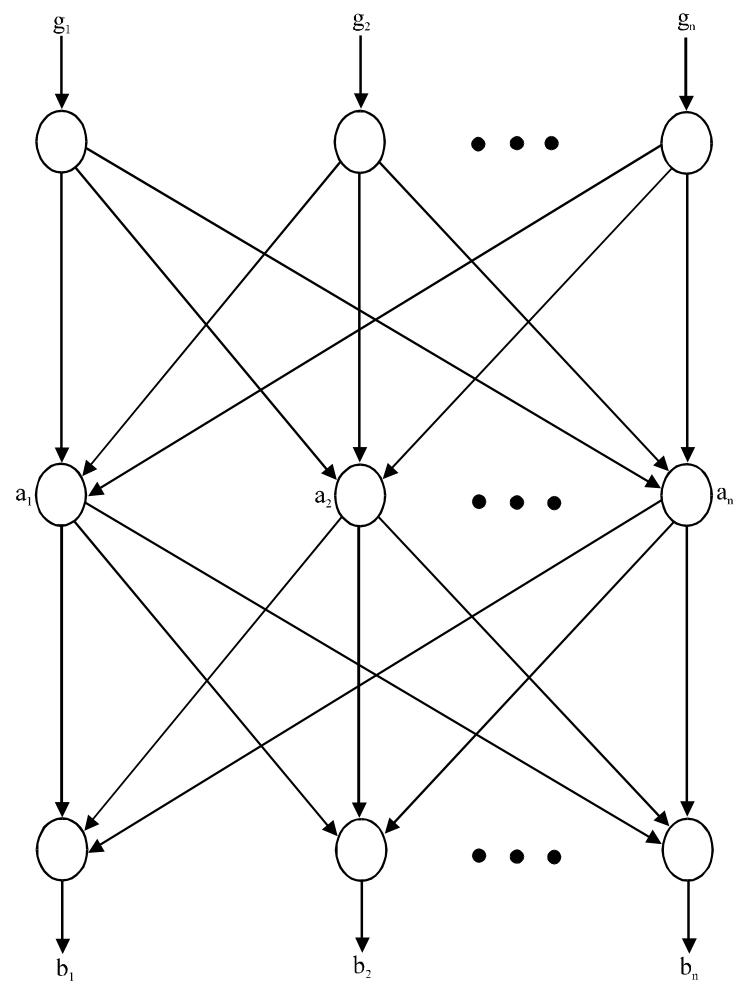

Fig. 1: The structure of FNN

parallel compute, distributed information storage, tolerant and self-study. Now FNN is applied in complex systems with high precision and efficiency.

Adding ANN method to FCE mentioned above it obtains new structure FNN shown as Fig. 1. There are the evaluation indexes $\left\{g_{1}, g_{2}, \ldots, g_{n}\right\}$ which scaled from the certain object. The neural network has three layers. The input layer has $\mathrm{n}$ neurons to accept $\mathrm{n}$ input signals $\left\{\mathrm{g}_{1}, \mathrm{~g}_{2}, \ldots, \mathrm{g}\right\}$. The hidden layer also has $\mathrm{n}$ neurons computed from input signals which are a fuzzy sub-collection $\left\{a_{1}, a_{2}, \ldots, a_{n}\right\} L$ of factor collection $U$. The output layer has $m$ neurons mapped a fuzzy sub-collection $\left\{\mathrm{b}_{1}, \mathrm{~b}_{2}, \ldots, \mathrm{b}_{\mathrm{n}}\right\}$ of the evaluation collection.

The fundamental types of gear of $\mathrm{CM}$ for marine electric impetus framework incorporate drive transformer, impetus converter and drive engine. These types of gear are while circle the association is commonly and affected by the outside condition. In this study, as indicated by the structure and operation rule of marine electric impetus framework joined the FNN and turn into the CM work process of marine electric drive framework, work process is appeared in Fig. 2.

According the real-time input data it evaluates, respectively the state of propulsion transformer and propulsion converter and propulsion motor combined environment factors at first, then evaluates synthetically

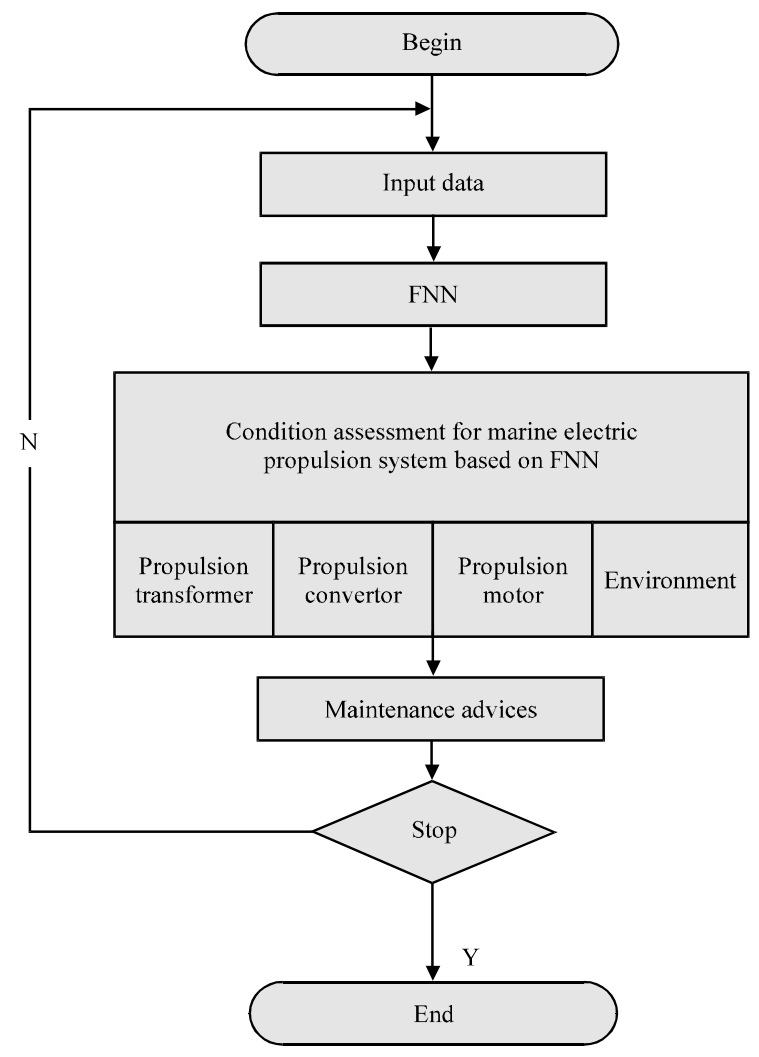

Fig. 2: Condition maintenance workflow of marine electric propulsion system

the system and obtains the score. The evaluation result is the scale of zero to one hundred score. The zero score is shown that the status index is near or over the attention value and it needs to maintenance.

While the marine electric propulsion system operation state good it means that all status values are away to the attention value and have no bad trends and no experience badness work state but few quality faults. Then the marine electric propulsion system maintenance can be on plan or delayed. While the marine electric propulsion system operation state normal it means that all status values are near to the attention value and have no visible badness trends and experience few badness work state and have quality faults. Then it may be priority to arrange maintenance according running condition.

While the marine electric impetus framework operation state sub-par it implies that all status esteems are close or achieve the consideration esteem and have obvious disagreeableness drifts and have disagreeableness work state records and have family quality issues. At that point it might organize upkeep soon. 


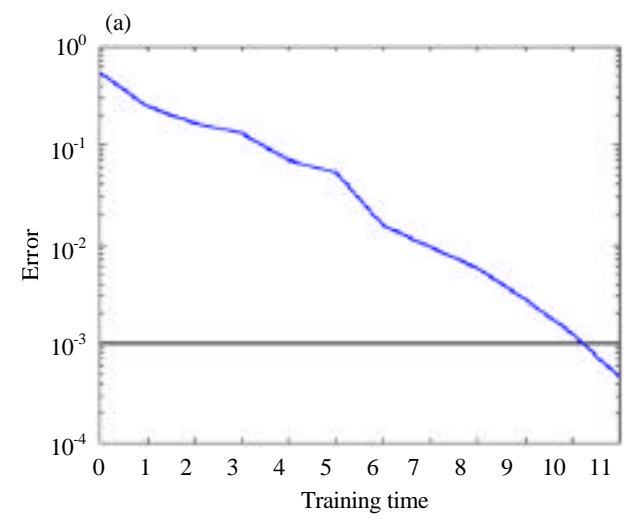

Fig. 3: a, b) Training curve

\section{RESULTS AND DISCUSSION}

Simulation: We study the simulation analysis of the CM model for the electric propulsion system of a certain ship. At first we evaluate devices based on FNN method according to the process of marine electric propulsion system CM shown in Fig. 2. Firstly, one assesses the propulsion transformer condition. The basic parameters of its transformer are given as follows: the rated power is $1450 \mathrm{KVA}$, the rated current is $1220 \mathrm{~A}$ input voltage is $660 \mathrm{~V}$, output voltage is $690 \mathrm{~V}$ and insulating grade level is $\mathrm{F}$.

Select component accumulation $U$ and assessment gathering $\mathrm{V}$ as howl: then characterized enrollment work utilizing semi-angle appropriated capacity and master tally technique forinstance the winding temperature has a place with the participation capacity of winding overheating is: the winding temperature dosage not have a place with temperature increase high and over-present and over-voltage, specifically, $0121314 \mathrm{r}=\mathrm{r}=\mathrm{r}=$. The winding temperature measurements not has a place with the participation capacity of typical state 1 and ordinary state 2 is: introduced FNN utilizing $\mathrm{R}$ above and prepared neural system with preparing tests as it got the preparation bend appeared as Fig. 3a. The non-instated preparing bend is appeared as outline $3 \mathrm{~b}$. It is clearly that FNN analyzed ANN the preparation speed enhanced around 6 times and furthermore has quick meeting.

\section{CONCLUSION}

Fuzzy Neural Network (FNN) strategy for condition upkeep of marine electric impetus framework is connected in the study. It spoke to the prevalence of FNN strate gy

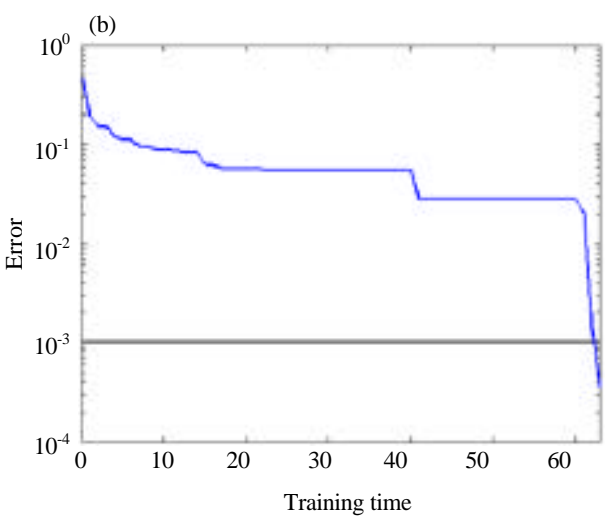

and utilizing FNN to set up the advance and actualize ventures of condition support of marine electric drive framework. Utilizing the specific ship's estimation information it acquired a model of condition support demonstrate with FNN. Reenactment utilizing MATLAB demonstrates that it can give high exactness and is reasonable for venture application.

\section{REFERENCES}

Bao, Y. and W. Shi, 2011. Research on operation simulation of ship electric propulsion system. Navigation China, 34: 34-38.

Chengguang, N.L.S.S.L. and K. Wenwen, 2012. Real-time condition assessment for power transformer. Electr. Eng., 10: 1-14.

Feng, S., B. Li, W. Chen, Y. Sun and Y. Cao et al., 2012. Analysis of CBM of transmission and transformation equipment. Heilongjiang Electric Power, 34: 223-225.

Lai, Y.J. and C.L. Hwang, 1992. Fuzzy Mathematical Programming: Methods and Applications. SpringerVerlag, Berlin, Germany, ISBN-13: 9783540560982 , Pages: 301.

Liao, R., H. Zheng, S. Grzybowski, L. Yang, Y. Zhang and Y. Liao, 2011. An integrated decision-making model for condition assessment of power transformers using fuzzy approach and evidential reasoning. IEEE Trans. Power Delivery, 26: 1111-1118.

Musheng, X., C. Jian and L. Rui, 2011. Assessment of the bridge technical state based on the BP neural net work. Trans. Sci. Technol., 5: 41-44.

Sethuramalingam, T.K. and B. Nagaraj, 2016. A proposed system of ship trajectory control using particle swarm optimization. Procedia Comput. Sci., 87: 294-299. 